\title{
Structural Evaluation of an Iron Oxalate Complex Layer Grown on an Ultra-smooth Sapphire (0001) Surface by a Wet Method
}

\author{
R. Harukia, ${ }^{\text {a,d }}$ O. Sakata ${ }^{\text {a,d }}$, T. Yamada ${ }^{\text {b,d }}$, K. Kanaizuka ${ }^{\text {b,d }}$, R. Makiura ${ }^{\text {b,d }}$, \\ Y. Akita ${ }^{c}$, M. Yoshimoto ${ }^{c}$, and H. Kitagawa ${ }^{\text {bd }}$ \\ aJapan Synchrotron Radiation Research Institute / SPring-8, Kouto, Sayo, Sayo, Hyogo 679-5198, Japan \\ Fax:81-(0)791-58-0830, e-mail: haruki@spring8.or.jp \\ ${ }^{\mathrm{b}}$ Department of Chemistry, Kyushu University, Hakozaki, Higashi-ku, Fukuoka, 812-8581, Japan \\ ${ }^{c}$ Materials and Structures Laboratory, Tokyo Institute of Technology, Nagatsuta 4259, Midori-ku, Yokohama, 226-8503, Japan \\ ${ }^{\mathrm{d} J S T-C R E S T, ~} 5$ Sanban-cho, Chiyoda-ku, Tokyo, 102-0075, Japan
}

\begin{abstract}
A crystallographic structure of an iron oxalate ultra-thin film grown on an ultra-smooth sapphire (0001) substrate was analyzed using synchrotron x-ray reflectivity and grazing incidence x-ray diffraction. The results show that the iron oxalate layer formed in crystal. In-plane diffraction measurements show the structure of the layer is random, and out-of-plane grazing incidence diffraction measurements show that the iron oxalate structure of the layer is affected by the step structure of sapphire surface, assumed to bond to the step edge.
\end{abstract}

Key words: iron oxalate, ultra-thin film, ultra-smooth sapphire, synchrotron $\mathrm{x}$-ray diffraction, $\mathrm{x}$-ray reflectivity

\section{INTRODUCTION}

A thin layer composed by metal complexes has been studied in order to apply to electronic devices and catalysts for fuel cell[1]. This system has been studied by many methods such as UV absorbance spectroscopy[2], variable-angle spectroscopic ellipsometry[2], and $x$-ray diffraction[3,4]. In ref. 4 , grazing incidence $x$-ray diffraction (GIXD) is applied to the metal complex layer with a thickness of $100-500 \mathrm{~nm}$ and compared with polycrystalline material. The metal complex layer with several $\mathrm{nm}$ in thickness is not yet studied by GIXD.

Although the molecules build up in same direction on the plane surface will be an useful nano-device, the in-plane structure of the molecules have not discussed in detail. Moreover those metal complexes should be arranged on the surface and the structure should be able to have controlled. GIXD with synchrotron $x$-ray is a powerful method to investigate structure in a several $\mathrm{nm}$ layer.

Iron cations with oxalic acid make iron oxalate dihydrate, which is generally known to have two structures. One is monoclinic, the other is orthorhombic[5]. In this paper, we have studied the structure of an iron oxalate ultra-thin layer $(<5 \mathrm{~nm})$ on a sapphire substrate. We used a single crystal sapphire substrate with an ultra-smooth surface to investigate simpler system. This sapphire substrate has a surface of a step structure[6].

\section{SAMPLE PREPARATION}

A single crystal sapphire substrate with ultra-smooth surface of $5 \times 7 \mathrm{~mm}^{2}$ was used[5]. The substrate was cleaned for 5 minutes using acetone and ethanol in turns and repeated twice. (A) The cleaned sapphire was immersed in a $0.10 \mathrm{mM}$ oxalic acid solution $(100 \mathrm{ml})$ for 12 hours at room temperature. After immersed, it was washed by distilled water and dried with argon gas. (B) And immersed in a $0.10 \mathrm{mM}$ iron sulfate solution (100 ml) for 12 hours at RT, and washed and dried. (A)-(B) was repeated 4 times in order to make 4-unit chain on the substrate.

\section{SYNCHROTRON X-RAY MEASUREMENTS}

Measurements were performed with a six-circle diffractometer at beamline BL13XU[7] for surface and interface structures, SPring-8. The experimental setup included a beamline $\mathrm{Si}(111)$ double crystal monochromator and mirrors. A wavelength used was 0.1 nm. A sample sat on the diffractometer, which was operated using an in-plane and out-of-plane geometry controlled through the spec software. The signals were measured by a Si PIN photo-diode detector, and a scintillation counter. The sample was kept in a kapton dome filled with $\mathrm{He}$ gas during the measurements. The measurements were performed at room temperature.

\section{RESULTS AND DISCUSSION}

4.1 X-ray Reflectivity Results

The X-ray reflectivity data from an iron oxalate layer on sapphire substrate is shown in Fig. 1 by a solid line.

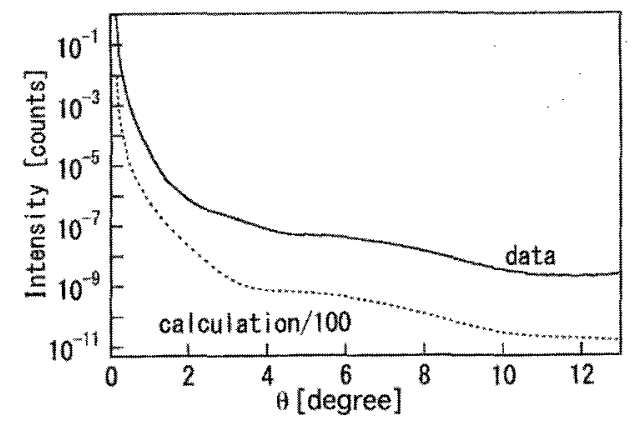

Fig. 1. The X-ray reflectivity data from the sample are shown by a solid line. Dotted line is a calculated result divided by 100 with a model with a single layer on a sapphire substrate. 
The data shows that a critical angle for total reflection is $0.185^{\circ}$. This is good agreement with the $0.1891^{\circ}$, the critical angle of a sapphire substrate. A model we used here is a single layer with roughness at the top and interface. A fitted curve shows that the results of the layer thickness, substrate roughness, and surface roughness are $0.41 \mathrm{~nm}, 0.066 \mathrm{~nm}, 0.00012 \mathrm{~nm}$, respectively. The obtained refractive index $\delta$ is $3.7 \times 10^{-6}$, and $\beta$ is $8.0 \times 10^{-8}$, for $1-\delta-i \beta$. The refractive index $\delta$ for iron oxalate in monoclinic system, is $3.07 \times 10^{-6}$, and $\beta$ is $6.75 \times 10^{-8}$. The obtained results are $20 \%$ larger than these values. The layer would be $20 \%$ denser than a bulk iron oxalate, if it is homogeneous along its depth. A value of density of a layer and values of top and interface roughness cannot separate in $\mathrm{x}$-ray reflectivity analysis. The fitting with other initial values may give other sets of parameters for density and roughness, but the result obtained for the layer thickness would not change basically.

The result that the layer thickness was $0.41 \mathrm{~nm}$, shows the fact that the layer is not a perfect single layer consisted with iron oxalate chains normal to the surface of the substrate. A unit of an iron oxalate dihydrate is longer than $0.41 \mathrm{~nm}$ and 4-unit-chain would be over 2 $\mathrm{nm}$ in length. The chains should be in parallel to the surface. The fitted result is largely deviated from the experimental data in the $1^{\circ}$ to $3^{\circ}$ and larger than $12^{\circ}$.

\subsection{Grazing Incidence X-ray Diffraction Results}

Grazing incidence $X$-ray diffraction profiles are shown in Fig. 2. The profiles were $2 \theta$ scan from $1^{\circ}$ to $50^{\circ}$ at the incidence angle $\theta$ of $0.1^{\circ}$, and azimuth angle $\phi$ fixed at $0^{\circ}$ and $90^{\circ}$. In all profiles, a peak at $12.3^{\circ}$ was observed. Around the $12.3^{\circ}$ peak, a broad peak was observed. These peaks were not observed in the data without a kapton dome around the sample. The broad peak intensity in the out-of-plane profiles is smaller than that in the in-plane profiles. Peaks are at $16.9^{\circ}$ and $41.1^{\circ}$ in the out-of-plane profile at $\phi=0$ whereas no peaks in the out-of-plane profile at $\phi=90^{\circ}$

\subsection{Discussion}

We will start from a structure prepared by the method we used here. The structure in this method after the sapphire substrate immersed in an oxalic acid solution is depicted in Fig. 3(a). After the substrate was washed by water and immersed in an iron sulfate solution, iron cations were assumed to be coordinated to oxalic acid, which is in Fig 3(b). These two processes were repeated to grow the iron oxalate chain. Fig. 3(c) depicts the chain structure after the procedure was repeated four times when it was grown perpendicular to the surface plane ideally. Water molecules between the chains are not depicted. In this method, the chain structure with iron cations and oxalic acids are assumed to grow on the substrate. The length between iron cations is $0.527 \mathrm{~nm}$ in a bulk iron oxalate of monoclinic system [3].

In the in-plane profile, the broad peak around $12.3^{\circ}$ has different shape compared to that in the out-of-plane profiles; that a peak at $10.9^{\circ}$ which indicates $0.527 \mathrm{~nm}$ is added to a broad peak observed in the out-of-plane. This is good agreement with that the reflectivity data shows that the iron oxalate chains grown on the substrate is parallel to the substrate surface, and they take any

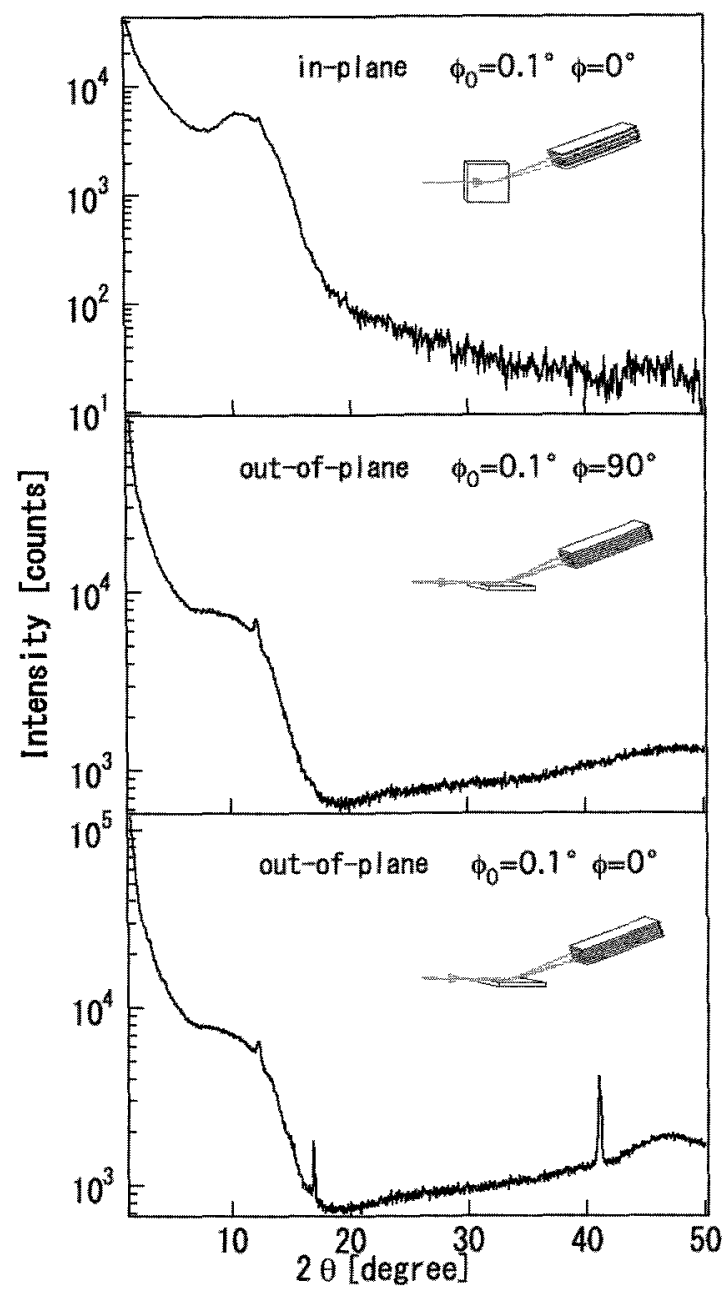

Fig. 2. Grazing incidence diffraction $2 \theta$ scan data at in-plane position with $\phi=0^{\circ}$, and at out-of-plane position with $\phi=0^{\circ}, 90^{\circ}$. The incidence $\mathrm{x}$-ray and the substrate with a Soller slit is depicted in the figures.

directions in-plane as shown in Fig. 3(d).

The peaks were observed in the out-of-plane profiles at $\phi=0^{\circ}$. In this layer-by-layer method, the distances between the chains were not controlled at all. In the case that the distance of the chains satisfies the condition, the chains will crystallize. This condition is not easily achieved when the bonding is strictly decided by the substrate crystal, in this case, sapphire single crystal c-face. This is the reason why there are no peaks observed in in-plane and out-of-plane $\phi=90^{\circ}$ direction profiles. Then we should introduce some reason to explain the peaks in the out-of-plane $\phi=0^{\circ}$ profile. It is described by the step structure the ultra-smooth sapphire substrate has[6]. The step structure generally has higher bonding ability than the terrace part. The iron oxalate may bond to the steps and crystallize. The peaks at $16.9^{\circ}$ and $41.1^{\circ}$ represent the distances between planes, $0.340 \mathrm{~nm}$ and $0.142 \mathrm{~nm}$, respectively. 
(a)

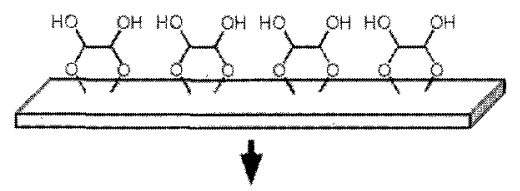

(b)

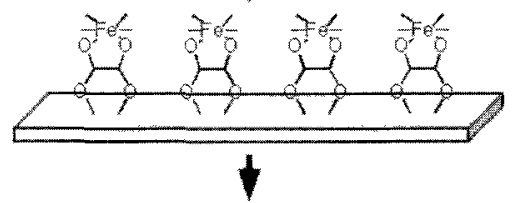

(c)

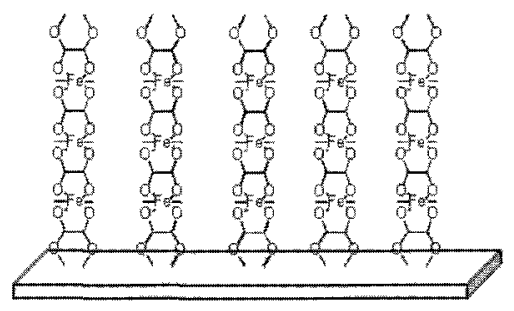

(d)

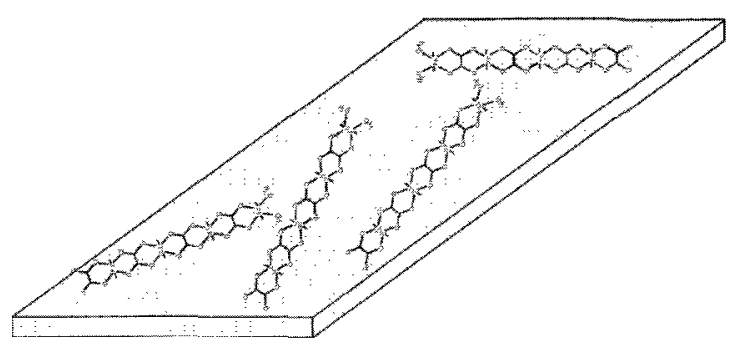

Fig. 3. (a) A bare sapphire substrate after immersed in an oxalic acid solution. (b) Iron atoms bonded to (a) after immersed in an iron sulfate solution. (c) Proposed chain structure of iron oxalate on a sapphire surface after (a) and (b) repeated 4 times. (d) The chains randomly placed on the substrate.

\section{SUMMARY}

An iron oxalate layer grown by layer-by-layer method on a sapphire substrate confirmed to take a crystal structure by $\mathrm{x}$-ray reflectivity and grazing incidence $x$-ray diffraction. The layer structure is highly affected by a sapphire substrate. The thickness of the iron oxalate layer on a single crystal sapphire c-face was measured by $x$-ray reflectivity. The experimental results show that the roughness of the surface of layer and the interface between layers and sapphire is low. This result shows good agreement with the model that the iron oxalate layers grow as we immerse in a solution of iron sulfoxide and oxalate solution in preparing this sample. On the other hand, there were no peaks observed in the in-plane profiles. This fact shows that the iron oxalate chain structure took any direction in-plane. Out-of-plane results show that the chains formed in crystal on the step structure of the sapphire surface. The chains also have a disordered structure. We infer from this result that the lattice parameters of iron oxalate and sapphire are totally different and iron oxalate layers cannot form a large crystal structure, and split into domain structures.

\section{ACKNOWLEDGEMENT}

The synchrotron radiation experiments were performed at SPring-8 with the approval of Japan Synchrotron Radiation Research Institute (JASRI). The measurements were also supported by JASRI under proposals No. 2007A1680, 2007B1899, and JST-CREST.

\section{REFERENCES}

[1] R. Bashyam, and P. Zelenay, Nature, 443, 63-66 (2006).

[2] L. Kosbar, C. Srinivasan, A. Afzali, T. Graham, M. Copel, and L. Krusin-Elbaum, Langmuir, 22, 7631-7638 (2006).

[3] A. A. Dakhel, Cryst. Res. Technol. 41, 68-71 (2006).

[4] S. Hermes, F. Schröder, R. Chelmowski, C. Wöll, and R. A. Fischer, J. Am. Chem. Soc., 127, 13744-13745, (2005).

[5] R. Deyrieux, A. Peneloux, Bull. Soc. Chim. Fr. 2675-2681 (1969).

[6] M. Yoshimoto, T. Maeda, T. Ohnishi, H. Koinuma, O. Ishiyama, M. Shinohara, M. Kubo, R. Miura and A. Miyamoto, Appl. Phys. Lett. 67, 2615-2617 (1995).

[7] O. Sakata, Y. Furukawa, S. Goto, T. Mochizuki, T. Uruga, K. Takeshita, H. Ohashi, T. Ohata, T. Matsushita, S. Takahashi, H. Tajiri, T. Ishikawa, M. Nakamura, M. Ito, K. Sumitani, T. Takahashi, T. Shimura, A. Saito, and M. Takahasi, Surf. Rev. Lett. 10, 543-547 (2003).

[8] Y. Tao, J. Am. Chem. Soc 115, 4350-4358 (1993).

(Recieved December 10, 2007 ; Accepted April 22, 2008) 